\title{
Crosstalk Cancellation in Digital Subscriber Lines Using Multidimensional Coordination
}

\author{
Minho Cheong and Yong-Hwan Lee \\ Seoul National University \\ Kwanak P. O. Box 34, Seoul 151-744, Korea \\ Tel: 82-2-880-8435, E-mail: mobilize@hanmail.net
}

\begin{abstract}
Digital Subscriber Lines (DSL) technology provides a transport of high-bit-rate digital information over the public telephone lines. Recently, a new technique has been proposed to increase the overall data rate by coordinating all the wires in the same DSL cable. However, this coordination method cannot be applied when the cable lines are used for different types of xDSL services. This paper proposes a multi-dimensionsal decision-feedback equalizer (M-D DFE) and precoding method that can simultaneously coordinate the lines in the time and user domain. A modified decision-feedback substitution is proposed by applying a generalized DFE (G-DFE) scheme into the two dimensional domain to increase the cable capacity. The performance of the proposed scheme is verified by computer simulation.
\end{abstract}

Keywords- DSL, cable coordination, multi-dimensional DFE/precoding

\section{INTRODUCTION}

Crosstalk, rather than additive noise, represents the major limitation in several multi-user digital communication systems. Currently, consecutive and suboptimum techniques are used to fight crosstalk [1-2]. Employing techniques that alleviate the impact of crosstalk holds the key in delivering further improvements in the performance of such systems.

The crosstalk problem has been previously studied in various contexts. In [3-4], multi-channel signal processing was introduced and the corresponding minimum mean square error (MMSE) linear equalizer was specified. In the absence of user coordination, wider than Nyquist transmitters have been shown to provide a performance advantage over Nyquist-limited transmitters [5-6]. Recently, Cioffi proposed a modulation scheme that can cancel out the FEXT (far-end crosstalk) by jointly processing the user signal at both the receiver and transmitter [7-8]. But, this coordination scheme cannot be applied to the cables where different DSL services are colocated and the NEXT (near-end crosstalk) is not negligible. To alleviate this problem, we propose a new cancellation scheme by using multi-dimensional DFE (MD DFE) and precoding with the coordinating structure at the central office.

Conventional coordination method is described in
Section II. Section III describes the proposed coordination method using multi-dimensional DFE. Section IV shows simulation results exhibiting the potential performance improvements for DSL applications. Finally, Section V provides the concluding remarks.

\section{CONVENTIONAL COORDINATION}

Consider a G-DFE structure [7-9] as shown in Figure 1, where $\mathbf{H}$ is the overall channel matrix in a DSL cable, A is a pre-distortion matrix derived by the matched filter and $\mathbf{u}$ is the pre-distorted data vector. The output of the matched filter can be represented as

$$
\mathbf{z}=\mathbf{R}_{f} \mathbf{u}+\mathbf{n}^{\prime}
$$

where $\mathbf{R}_{f}=\mathbf{A}^{*} \mathbf{H}^{*} \mathbf{H A}$ and $\mathbf{n}^{\prime}=\mathbf{A}^{*} \mathbf{H}^{*} \mathbf{n}$. Note that the covariance of noise $\mathbf{n}$ is $\mathbf{R}_{n n}=\mathbf{R}_{f}$. Applying the Cholesky factorization to $\mathbf{R}_{f}$, we have

$$
\mathbf{R}_{f}=\mathbf{G}^{*} \mathbf{S}_{o} \mathbf{G}
$$

where $\mathbf{S}_{o}$ is a diagonal matrix with positive elements and $\mathbf{G}$ is a monic upper triangular matrix. Processing the received vector $\mathbf{z}$ by $\mathbf{S}_{o}^{-1} \mathbf{G}^{-*}$ gives

$$
\mathbf{S}_{o}^{-1} \mathbf{G}^{-*} \mathbf{z}=\mathbf{G u}+\mathbf{S}_{o}^{-1} \mathbf{G}^{-*} \mathbf{n}^{\prime}=\mathbf{G u}+\mathbf{e}
$$

Since $\mathbf{G}$ is an upper triangular matrix and error $\mathbf{e}$ has uncorrelated components, the input $\mathbf{u}$ can be recovered by back-substitution combined with symbol-by-symbol detection. Thus, a decision feedback structure can be constructed with feedforward filter $\mathbf{S}_{o}^{-1} \mathbf{G}^{-*}$ and feedback filter $(\mathbf{I}-\mathbf{G})$ as in Figure 1. The transmit filter in the GDFE structure can be defined as

$$
\mathbf{A}=\mathbf{Q}_{m I D F T} \mathbf{P} .
$$

where $\mathbf{Q}_{\text {mIDFT }}$ and $\mathbf{P}$ denotes an $L-\mathrm{D}$ expansion of DFT matrix $\mathbf{Q}_{I D F T}$ with $N$ tones and a permutation matrix, respectively. $\mathbf{R}_{f}$ can be factored as

$$
\mathbf{R}_{f}=\mathbf{P}^{*} \mathbf{Q}_{m D F T} \mathbf{H}^{*} \mathbf{H} \mathbf{Q}_{m I D F T} \mathbf{P}
$$

Recalling that $\mathbf{H}_{i, j}$ of matrix $\mathbf{H}$ are circulant, we have $\mathbf{H}_{i, j}=\mathbf{Q}_{I D F T} \boldsymbol{\Lambda}_{i, j} \mathbf{Q}_{D F T}$ where $\boldsymbol{\Lambda}_{i, j}$ is a diagonal matrix. Thus, (5) can be rewritten as

$$
\mathbf{R}_{f}=\mathbf{P}^{*} \Lambda^{*} \Lambda \mathbf{P}
$$




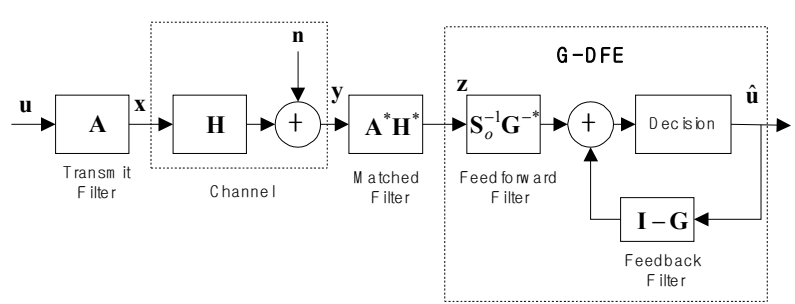

Figure 1. G-DFE structure.

where $\boldsymbol{\Lambda}_{i, j}$ is the $(i, j)$-th element of $\boldsymbol{\Lambda}$. The reordering $\mathbf{R}_{f}$ by $\mathbf{P}$ results in a block diagonal matrix

$$
\mathbf{R}_{f}=\operatorname{diag}\left(\mathbf{R}_{f, 1}, \mathbf{R}_{f, 2}, \ldots, \mathbf{R}_{f, N}\right)
$$

where $\mathbf{R}_{f, i}, i=1, \ldots, N$ is an $(L \times L)$-D matrix. The Cholesky factorization of $\mathbf{R}_{f}$ is represented as

$\mathbf{R}_{f}=\operatorname{diag}\left(\mathbf{G}_{1}^{*} \mathbf{S}_{o, 1} \mathbf{G}_{1}, \mathbf{G}_{2}^{*} \mathbf{S}_{o, 2} \mathbf{G}_{2}, \ldots, \mathbf{G}_{N}^{*} \mathbf{S}_{o, N} \mathbf{G}_{N}\right)$

Then, we have the following decomposition

$$
\mathbf{P}^{*} \mathbf{\Lambda} \mathbf{P}=\operatorname{diag}\left(\mathbf{Q}_{1} \mathbf{R}_{1}, \mathbf{Q}_{2} \mathbf{R}_{2}, \ldots, \mathbf{Q}_{N} \mathbf{R}_{N}\right)
$$

where $\mathbf{Q}_{i}, i=1, \ldots, N$ is an $(L \times L)$-D unitary matrix, and $\mathbf{R}_{i}, i=1, \ldots N$ is an $(L \times L)-\mathrm{D}$ upper triangular matrix. Simple substitution proves that $\mathbf{R}_{i}=\mathbf{S}_{o, i}^{\frac{1}{2}} \mathbf{G}_{i}$.

Consider the G-DFE receiver components. The feedback filter $\mathbf{G}=\operatorname{diag}\left(\mathbf{G}_{1}, \mathbf{G}_{2}, \ldots, \mathbf{G}_{N}\right)$ can be broken into $N$ independent feedback filters, each of which operates at each tone. The combination of the matched filter and feedforward filter is

$$
\begin{aligned}
\mathbf{S}_{o}^{-1} \mathbf{G}^{-*} \mathbf{A}^{*} \mathbf{H}^{*} & =\mathbf{S}_{o}^{-1} \mathbf{G}^{-*} \mathbf{P}^{*} \boldsymbol{\Lambda}^{*} \mathbf{Q}_{m D F T} \\
& =\mathbf{S}_{o}^{-1} \mathbf{G}^{-*} \mathbf{P}^{*} \boldsymbol{\Lambda}^{*} \mathbf{P} \mathbf{P}^{*} \mathbf{Q}_{m D F T}
\end{aligned}
$$

From (9), we have

$$
\begin{aligned}
\mathbf{S}_{o}^{-1} \mathbf{G}^{-*} \mathbf{A}^{*} \mathbf{H}^{*} & =\mathbf{S}_{o}^{-1} \mathbf{G}^{-*} \operatorname{diag}\left(\mathbf{R}_{1}^{*} \mathbf{Q}_{1}^{*}, \mathbf{R}_{2}^{*} \mathbf{Q}_{2}^{*}, \ldots, \mathbf{R}_{N}^{*} \mathbf{Q}_{N}^{*}\right) \mathbf{P}^{*} \mathbf{Q}_{m D F T} \\
= & \mathbf{S}_{o}^{-1} \mathbf{G}^{-*} \operatorname{diag}\left(\mathbf{G}_{1}^{*} \mathbf{S}_{o, 1}^{\frac{1}{2}}, \mathbf{G}_{2}^{*} \mathbf{S}_{o, 2}^{\frac{1}{2}}, \ldots, \mathbf{G}_{N}^{*} \mathbf{S}_{o, N}^{\frac{1}{2}}\right) \\
& \cdot \operatorname{diag}\left(\mathbf{Q}_{1}^{*}, \mathbf{Q}_{2}^{*}, \ldots, \mathbf{Q}_{N}^{*}\right) \mathbf{P}^{*} \mathbf{Q}_{m D F T} \\
& =\operatorname{diag}\left(\mathbf{S}_{o, 1}^{-\frac{1}{2}}, \mathbf{S}_{o, 2}^{-\frac{1}{2}}, \ldots, \mathbf{S}_{o, N}^{-\frac{1}{2}}\right) \cdot \operatorname{diag}\left(\mathbf{Q}_{1}^{*}, \mathbf{Q}_{2}^{*}, \ldots \mathbf{Q}_{N}^{*}\right) \mathbf{P} \mathbf{Q}_{m D F T}
\end{aligned}
$$

\section{MULTIDIMENSIONAL COORDINATION}

Unless the DSL signal is modulated by MIMO DMT, it cannot be decomposed into one-tap (scalar) representation for each tone. A single-carrier DSL signal can be decomposed into a per-tone multi-tap equalizer structure.
To describe the single-carrier model, we consider the transmission of successive symbols of a user $X_{1: N}^{(t)}$ at $t=k-1, k, k+1$, where the $k$-th symbol is the desired one, the previous and next symbol are used to include the interference from the neighboring symbols. The received signal can be represented as

$$
\begin{aligned}
& \overbrace{\left[\begin{array}{c}
y_{k s+\nu-T+2} \\
\ddots \\
y_{(k+1) s}
\end{array}\right]}^{\mathbf{v}}\left[\mathbf{O}_{(1)}\left|\begin{array}{ccc}
\mathbf{h} & 0 & \cdots \\
\ddots & \ddots & \\
0 & \cdots & \mathbf{h}
\end{array}\right| \mathbf{\mathbf { O } _ { ( 2 ) }}\right] \cdot\left[\begin{array}{lll}
\mathbf{T} & \mathbf{O} & \mathbf{0} \\
\mathbf{O} & \mathbf{T} & \mathbf{0} \\
\mathbf{O} & \mathbf{O} & \mathbf{T}
\end{array}\right] \cdot\left[\begin{array}{ccc}
Q_{I D F T} & \mathbf{0} & \mathbf{0} \\
\mathbf{O} & Q_{I D F F} & \mathbf{0} \\
\mathbf{O} & \mathbf{O} & Q_{I D F T}
\end{array}\right] \cdot \overbrace{\left[\begin{array}{l}
X_{1: N}^{(k-1)} \\
X_{1: N}^{(k)} \\
X_{1: N}^{(k+1)}
\end{array}\right]}^{\mathbf{x}} \\
& +\overbrace{\left[\begin{array}{c}
n_{k s+v-T+2} \\
\vdots \\
n_{(k+1) s}
\end{array}\right]}^{\mathrm{n}} \\
& =\mathbf{H} \cdot \mathbf{X}+\mathbf{n}
\end{aligned}
$$
$(N+T-1) \times(N+v-K)$-D zero matrix, respectively. $v$ denotes the length of the guard interval of the far-end signal, $s=W+v$ the length of a single-carrier symbol and $\mathbf{h}=\left[h_{L} \cdots h_{0} \cdots h_{-K}\right]$ the channel impulse response in reverse order, and

$$
\mathbf{T}=\left[\begin{array}{l|l}
\mathbf{O} & \mathbf{I}_{\mathbf{v}} \\
\hline \mathbf{I}_{N}
\end{array}\right]
$$

Here, $\mathbf{I}_{N}$ matrix is an $(N \times N)$-D identity matrix.

Each receiver can be represented as a building block in (14). The received signal is detected by

$$
\left[\begin{array}{c}
\hat{X}_{1}^{(k)} \\
\vdots \\
\hat{X}_{N}^{(k)}
\end{array}\right]=\left[\begin{array}{ccc}
D_{1} & 0 & \cdots \\
0 & \ddots & 0 \\
\vdots & 0 & D_{N}
\end{array}\right] \cdot \overbrace{Q_{D F T} \cdot(\mathbf{Y} \cdot \mathbf{w})}^{1 F F T}
$$

where $\mathbf{w}=\left[w_{0} w_{1} \cdots w_{T-1}\right]^{T}$ denotes the real coefficient of the $T$-tap TEQ, $D_{i}$ is the 1-tap complex FEQ at the $i$-th tone, $\mathbf{Y}$ an $(N \times N)$ Toeplitz matrix containing the received signal, and

$$
\mathbf{Y}=\left[\begin{array}{cccc}
y_{k s+v+1} & y_{k s+v} & \cdots & y_{k s+v-T+2} \\
y_{k s+v+2} & y_{k s+v+1} & \cdots & y_{k s+v-T+3} \\
& \ddots & \ddots & \\
y_{k s+v+1} & y_{(k+1) s-1} & \cdots & y_{(k+1) s-T+1}
\end{array}\right]
$$

Our approach is to transfer the single-carrier filtering operation into the frequency domain (i.e., after the DFTdemodulation). At the $i$-th tone, we can rewrite the above operation

$X_{i}^{(k)}=D_{i} \cdot \operatorname{row}_{i}\left(F_{N}\right) \cdot(\mathbf{Y w})=\operatorname{row}_{i} \underbrace{\left(Q_{D F T} \cdot \mathbf{Y}\right)}_{T F F T s} \cdot \underbrace{\mathbf{w} \cdot D_{i}}_{\mathbf{w}_{i}}$ 
where $\operatorname{row}_{i}[\cdot]$ denotes the $i$-th row of its matrix. By putting $D_{i}$ to the right, one has $\mathbf{w} \cdot D_{i}=\left(w_{i}\right)_{T \times 1}$ which is a (complex) $T$-tap FEQ at the $i$-th tone. We can allow each tone to have its own optimum $T$-tap FEQ $\mathbf{w}_{i}$. Thus, the DMT receiver needs a multi-tap FEQ equalizer for each tone to receive the single-carrier modulated signal.

The signal in the coordinated receiver can be represented as a one-tap or multi-tap delay-line symbol for each tone. It is possible to analyze the MIMO channel in the coordinated receiver in the time and user domain. After 2-D Cholesky factorization, the DFE can be processed as shown in Figure 2, where circular marks denote the symbol interfering the delayed symbols and other users, and arrow lines represent possible consecutive substitution to cancel the ISI and crosstalks. Thus, we can devise a novel coordinating scheme with the use of two dimensional G-DFE (i.e., concatenated DFE). Sequential operation for this cancellation scheme can be given by

$$
\begin{aligned}
& \left(\widehat{S}_{i, n}\right)_{k}=\operatorname{dec}\left[\frac{1}{r_{k, k}^{i, n}}\left(\tilde{\mathbf{Z}}_{i, n}\right)_{k}-\sum_{j=n+1}^{M} \frac{r_{k, j}^{i, n}}{r_{k, k}^{i, n}}\left(\widehat{S}_{i, j}\right)_{k}\right], n=T, T-1, \ldots, 1 \\
& \left(\mathbf{U}_{n, k}\right)_{i}=\operatorname{dec}\left[\frac{1}{r_{k, k}^{i}}\left(\hat{S}_{i}\right)_{k}-\sum_{j=k+1}^{L} \frac{r_{k, j}^{i}}{r_{k, k}^{i}}\left(\hat{\mathbf{U}}_{n, j}\right)_{i}\right], k=L, L-1, \ldots, 1
\end{aligned}
$$

where $\operatorname{dec}[\cdot]$ denotes the decoding operator, $r_{k, j}^{i, n}$ represents the estimated crossalk between user $k$ and $j$ and intersymbol interference between symbol $i$ and $n$, and $\left(\hat{S}_{i, n}\right)_{k}$ denotes the intermediate decoded samples only after the G-DFE in the user domain. At each timing instant, we first decode the symbol employing a user domain G-DFE, then obtain ISI-free symbol by using the G-DFE in the time domain with several intermediate samples $\left(\hat{S}_{i, n}\right)_{k}$.

The concatenated DFE has a performance enhancement over 1-D Cioffi's G-DFE structure. The proposed scheme can cancel out the crosstalk at the very timing instant of the received symbol. However, this scheme cannot suppress the crosstalk which is not timing-aligned to the received symbol.

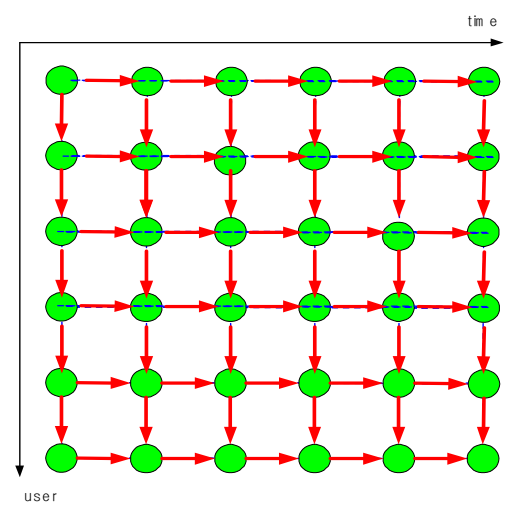

Figure 2. Sequential substitution of the concatenated DFE.
In order to suppress crosstalking symbols which are distant multi-symbols from the detected symbol, we propose a scheme called multi-dimensional (M-D) DFE. It transforms the consecutive substitution in the G-DFE into a 2-D MIMO processor as shown in Figure 3.

In Figure 3, for diagonal channel analysis, it is also required to decompose the MIMO channel which is timing-misaligned by users. The proposed M-D DFE can simultaneously suppress the ISI and crosstalk by sequentially processing as (19), for $k=L, L-1, \ldots, 1 \quad n=M, M-1, \ldots, 1$

$$
\left(\mathbf{U}_{n, k}\right)_{i}=\operatorname{dec}\left[\frac{1}{r_{k, k}^{i}}\left(\tilde{\mathbf{Z}}_{i}\right)_{k}-\sum_{l=n+1}^{M} \sum_{j=k+1}^{L} \frac{r_{k, j}^{i}}{r_{k, k}^{i}}\left(\hat{\mathbf{U}}_{l, j}\right)_{i}\right]
$$

where multiple summations represent wave-like 2-D feedback operations.

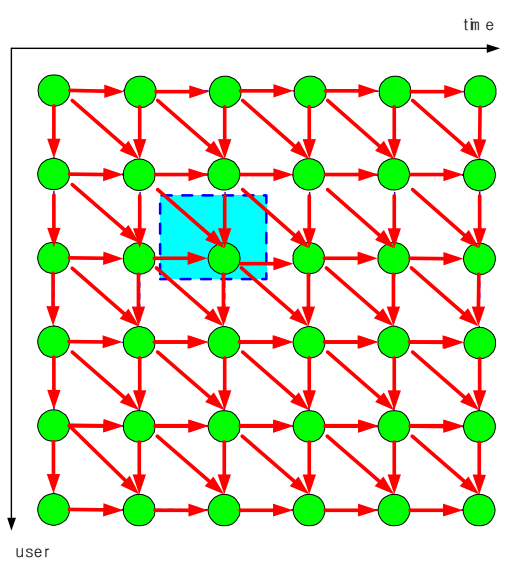

Figure 3. Sequential substitution of the M-D DFE.

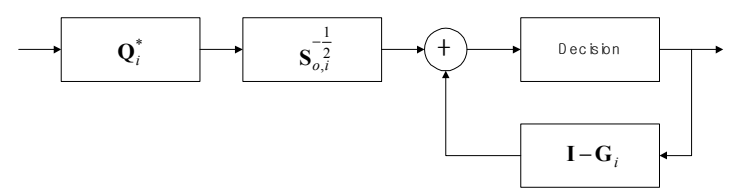

Figure. 4. Canceller block at the coordinated receiver.

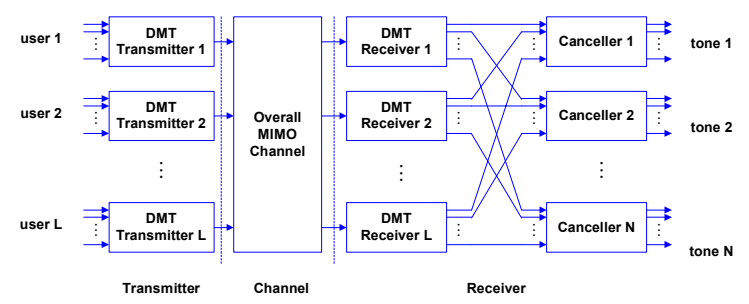

Figure 5. Tone-by-tone coordination using the proposed M-D DFE.

The M-D DFE described above per tone can be shown in Figure 4. Figure 5 depicts the overall coordination structure in tone-by-tone using the concatenated DFE or the M-D DFE. The single-carrier signal can be transmitted 
using this multi-tone structure. In this case, a guard interval is required within the overhead duration defined in the HDSL or SDSL specifications [1].

When a coordinated scheme is employed in the central office, MIMO channel can be analyzed (or Cholesky factorized) at the coordinated transmitter in the time and user domain, too. With 2-D Cholesky factorization, the consecutive substitution can be done as shown in Figure 2. The sequential substitution can be done as

$$
\begin{aligned}
& \left(\mathbf{U}_{n, k}\right)_{i}=\Gamma_{M}\left[\frac{1}{r_{k, k}^{i}}\left(\widehat{S}_{i}\right)_{k}-\sum_{j=k+1}^{L} \frac{r_{k, j}^{i}}{r_{k, k}^{i}}\left(\hat{\mathbf{U}}_{n, j}\right)_{i}\right], k=L, L-1, \ldots, 1 \\
& \left(\hat{S}_{i, n}\right)_{k}=\Gamma_{M}\left[\frac{1}{r_{k, k}^{i, n}}\left(\tilde{\mathbf{Z}}_{i, n}\right)_{k}-\sum_{j=n+1}^{M} \frac{r_{k, j}^{i, n}}{r_{k, k}^{i, n}}\left(\hat{S}_{i, j}\right)_{k}\right], n=M, M-1, \ldots, 1
\end{aligned}
$$

where $\Gamma_{M}[\cdot]$ denotes the modulo operator in the THprecoding, $r_{k, j}^{i, n}$ represents the crosstalking/intersymbol function estimated in [6], and $\left(\widehat{S}_{i, n}\right)_{k}$ denotes intermediately precoded samples only after the timedomain precoding. Similar to the coordinated receiver, the signal is first precoded in the time-domain, and in the user domain, becoming a purposely crosstalk-contaminated signal. The proposed M-D precoder simultaneously suppress the ISI and crosstalk by sequential processing as

$$
\left(\mathbf{U}_{n, k}\right)_{i}=\Gamma_{M}\left[\frac{1}{r_{k, k}^{i}}\left(\tilde{\mathbf{Z}}_{i}\right)_{k}-\sum_{l=n+1}^{M} \sum_{j=k+1}^{L} \frac{r_{k, j}^{i}}{r_{k, k}^{i}}\left(\hat{\mathbf{U}}_{l, j}\right)_{i}\right], k=L, \ldots, 1 \quad n=M, \ldots, 1
$$

The M-D precoder described above per tone can be shown in Figure 6. Figure 7 depicts the overall coordinated structure using the concatenated precoder or M-D precoder.

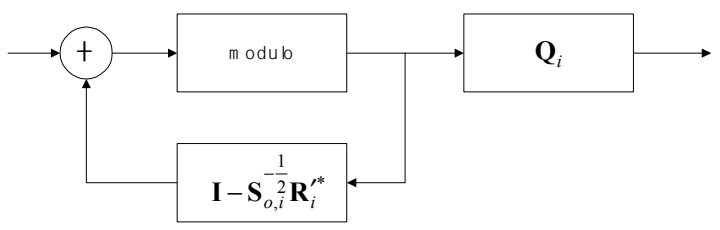

Figure. 6. Precoder block at the coordinated transmitter.

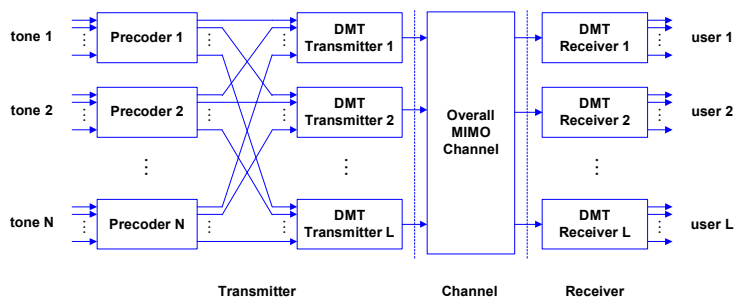

Figure 7. Tone-by-tone coordination using the proposed M-D precoding.

\section{SiMULATION RESULTS}

The proposed scheme can provide performance improvement of DSL transmission, where the NEXT and FEXT are the primary impairment factors. We consider VDSL [10] with a bandwidth of up to $20 \mathrm{MHz}$, yielding severe NEXT from several single-carrier schemes. The twisted pairs belonging to a single cable usually meet at the central office (CO) in the fiber-to-the-exchange (FTTEx) or remotely deployed optical network unit (ONU) in fiber-to-the-cabinet (FTTC) [10]. The simulation parameters shown in Table 1 are mainly taken from [5] and the DMT specifications are determined to keep backwards compatibility with the ADSL.

Figure 8 depicts the VDSL data rates achievable by the proposed multi-dimensional schemes in the downstream transmission. For comparison, the performance of Cioffi's scheme [7] is also depicted. It can be seen that the analysis agrees well with simulation results and that the M-D DFE can support higher data rate. The rate improvement is considerable when the loop length is less than 2000 feet, where the NEXT is dominant. It can also be seen that the proposed scheme can extend the loop length for a given data rate requirement. For example, the upstream transmission at $40 \mathrm{Mbps}$ is possible within the length of 1200 feet with the use of Cioffi's scheme, but it can be extended up to 2000 feet with the use of the proposed scheme.

Figure 9 depicts the ADSL data rate achievable in the downstream transmission with the use of the proposed scheme. The proposed scheme is quite effective in the loop with a short distance, yielding the throughput pretty much closer to the optimum one.

\section{CONCLUSIONS}

In this paper, we have proposed a new modulation scheme called M-D DFE and M-D precoding. The proposed coordinating scheme can cancel out the NEXT and FEXT either at the transmitter or receiver. The proposed scheme can be interpreted as a concatenated DFE and generalized M-D DFE with successive cancellation or precoding.

The performance of the proposed scheme is verified by computer simulation when applied to DSL systems. Simulation results show that the proposed scheme is quite efficient in NEXT-dominant environments, making it quite applicable to real DSL systems.

The proposed scheme requires computational burden only at the central office. There is a further possibility to reduce computational burden by detecting whether a line in the cable is busy or idle. In Korea, it is reported that only $30 \%$ of lines in a cable are busy usually. 
Table 1. Simulation parameters.

\begin{tabular}{|c|c|}
\hline Number of DMT tones & 4096 \\
\hline Tone Width & $4.3125 \mathrm{KHz}$ \\
\hline Symbol Rate & $4 \mathrm{KHz}$ \\
\hline Noise Margin & $6 \mathrm{~dB}$ \\
\hline Required BER & $<10^{-7}$ \\
\hline $\begin{array}{c}\text { Number of self-FEXT } \\
\text { Disturbers }\end{array}$ & 24 \\
\hline Maximum Bit Capacity & 15 \\
\hline Cable Type & 26-Gauge $(0.4 \mathrm{~mm})$ \\
\hline Source/Load Resistance & 100 Ohm \\
\hline Power Spectral Density Mask & As specified in [5] \\
\hline
\end{tabular}

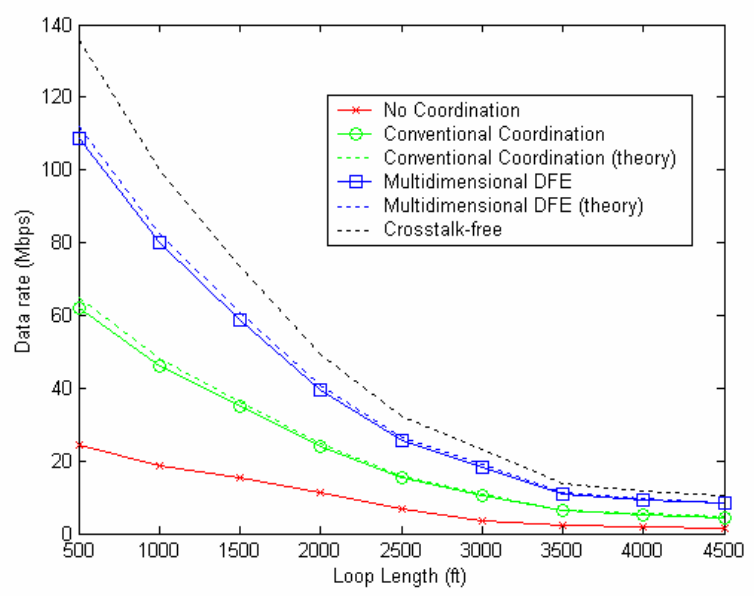

Figure 8. VDSL data rates.

(25 DMT VDSL, 5 SC VDSL, 5 T1, 10 HDSL, and 5 ISDN services are in a DSL cable)

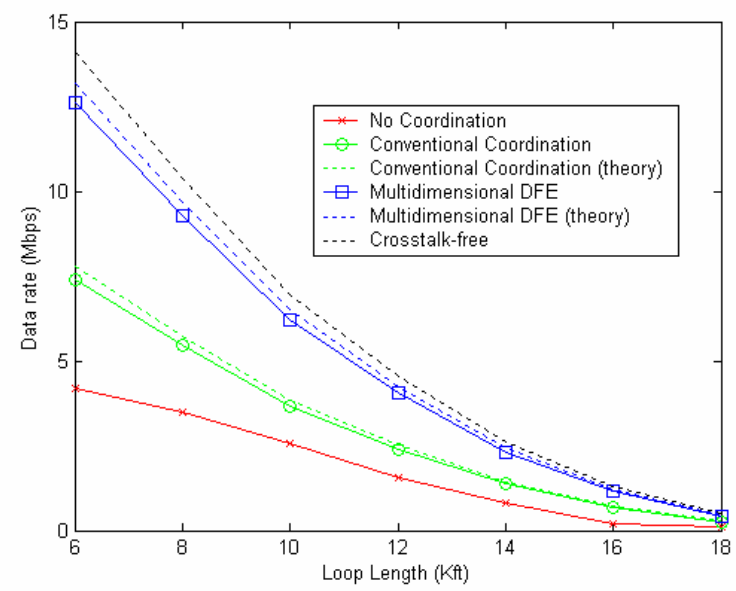

Figure 9. ADSL data rates.

(25 DMT ADSL, 10 HDSL, and 15 ISDN services are in a DSL cable)

\section{REFERENCES}

[1] T. Starr, J. M. Cioffi and P. Silverman, Understanding Digital Subscriber Line Technology, Prentice Hall, 1998.

[2] J. W. Cook, "The Noise and Crosstalk Environment for ADSL and VDSL systems", IEEE Commun. Magazine, May 1999, pp. 73-78.

[3] B. R. Peterson and D. D. Falconer, "Minimum Mean Square Equalization in Cyclostationary and Stationary Interference Analysis and Subscriber Line Calculations", IEEE J. on Selected Areas in Commun., Vol. 9, No. 6, Aug. 1991, pp. 931-940.

[4] M. Abdulrahman and D. D. Falconer, "Cyclostationary Crosstalk Suppression by Decision Feedback Equalization on Digital Subscriber Loops", IEEE J. on Selected Areas in Commun., Vol. 10, No. 3, Apr. 1992, pp. 640-649.

[5] ITU Recommendations G.992.1, Asymmetric Digital Subscriber Line (DSL) Transceivers, Jun. 1999.

[6] C. H. Zeng, C. Aldana, A. Salvekar and J. M. Cioffi, "Crosstalk Identification in xDSL Systems", IEEE J. on Selected Areas in Commun., Aug. 2001, pp. 14881496.

[7] G. Ginis and J.M. Cioffi, "Vectored Transmission for Digital Subscriber Line Systems," IEEE J. on Selected Areas in Commun., Vol. 20, No. 6, June 2002.

[8] G. Ginis and J.M. Cioffi, "A Multi-user Precoding Scheme achieving Crosstalk Cancellation with Application to DSL Systems," Proc. of the 34th Asilomar Conf., Pacific Grove, pp. 1627-1631, October 2000.

[9] J. M. Cioffi and G. D. Forney, "Generalized DecisionFeedback Equalization for Packet Transmission with ISI and Gaussian Noise," Chapter 4 of Communication, Computation, Control and Signal Processing, Kluwer, 1997.

[10] J. Bingham, ADSL, VDSL and Multicarrier Modulation, Wiley-Interscience Pub., 2000. 